EPJ Web of Conferences 71, 00066 (2014)

DOI: 10.1051/epjconf/20147100066

(C) Owned by the authors, published by EDP Sciences, 2014

\title{
Search for exotic phenomena at the CMS
}

\author{
Małgorzata Kazana ${ }^{1, a}$ on behalf of the CMS Collaboration \\ ${ }^{1}$ National Centre for Nuclear Studies, Hoża 69, Warsaw, Poland
}

\begin{abstract}
Signs of physics beyond the Standard Model are widely searched for in protonproton collisions at the Large Hadron Collider (LHC). We review results of exotic physics searches based on $20 \mathrm{fb}^{-1}$ of data collected in 2012 by the Compact Muon Solenoid (CMS) detector at a center-of-mass energy of $8 \mathrm{TeV}$. Selected benchmark analyses are presented, including searches in topologies with leptons, photons and jets, as well as a description of techniques to identify the production of exotic objects such as massive long-lived charged particles. No statistically-significant excess of events is observed in the data, therefore results are presented in terms of exclusion limits on the mass and the production cross section of hypothetical particles.
\end{abstract}

\section{Introduction}

The remaining unsolved puzzles of the Universe are the motivation for the LHC experiments to search for signs of New Physics.

The Standard Model (SM) of particle physics describing elementary particles and their interactions is very successful theory, which provides precise predictions for experimental measurements. Unfortunately, in the SM, several fundamental problems cannot be solved on the basis of first principles. For instance, within the SM, there is no candidate for the Dark Matter (DM), the source of matterantimatter asymmetry in quark flavour mixing is insufficient to account for the cosmic asymmetry, and the gravitational interaction is neglected. These and other issues are attempted to be resolved by theories Beyond the Standard Model (BSM). Supersymmetry [1] (SUSY) is proposed as a simple and elegant extension of the SM. Other theoretical approaches providing solutions to some of the problems mentioned above, and often offering a rich phenomenology, are grouped under the term exotic physics, or exotica, which in the CMS [2] Collaboration convention designates all the BSM physics except for SUSY. Exotica includes models with extra dimensions, new gauge bosons, black holes, leptoquarks and other models with unusual signatures as, for instance, delayed signals, kinked tracks or wide jets, and more.

In this article, we present a review of selected exotica searches in the CMS, based on the data collected at the Large Hadron Collider (LHC) at a center-of-mass energy of $8 \mathrm{TeV}$, and corresponding to 12 to $20 \mathrm{fb}^{-1}$ of integrated luminosity.

\footnotetext{
ae-mail: Malgorzata.Kazana@cern.ch
} 


\section{New Resonances}

Many models describing possible BSM physics predict the existence of resonances at the $\mathrm{TeV}$ mass scale. New resonances can couple to a pair of SM particles, leptons, quarks or pair of $\mathrm{W}$ and Z bosons. The resonance's signal is a peak on the tail of the mass invariant distribution of a given pair. This signature is clean, but experimentally challenging. It require a very good understanding of detector effects (efficiencies, uncertainties, trigger) and dedicated techniques to reconstruct and properly identify SM particles with high transverse momentum.

\subsection{Dilepton Resonances}

A search for narrow high-mass resonances $Z^{\prime}$ decaying to electron or muon pairs has been performed using $8 \mathrm{TeV}$ pp collision data collected by the CMS experiment [3]. The dimuon event sample corresponds to an integrated luminosity of $20.6 \mathrm{fb}^{-1}$, while the dielectron event sample corresponds to 19.6 $\mathrm{fb}^{-1}$, as determined by the different performance requirements for the various subdetectors.
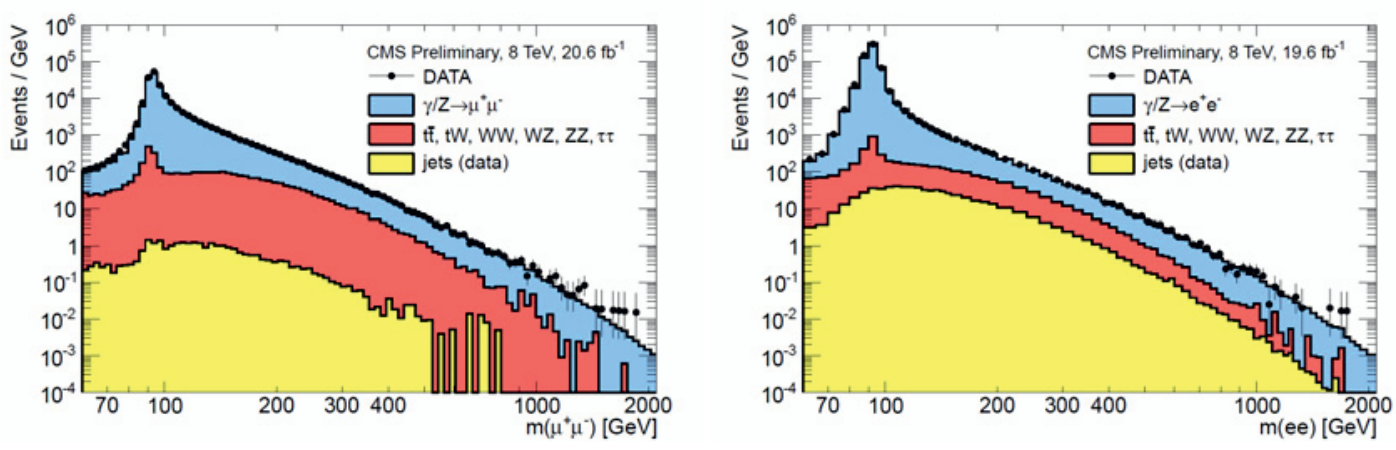

Figure 1. The invariant mass spectrum of $\mu^{+} \mu^{-}$(left) and $e e$ (right) events. The points with error bars represent the data. The histograms show the expectations from SM processes and the multijet backgrounds. Multijet backgrounds contain at least one jet that has been misreconstructed as a lepton.

Dilepton events are selected by a double electron trigger requiring two clusters in the ECAL with transverse energy $E_{T}>33 \mathrm{GeV}$ or collected using a trigger requiring the presence of at least one muon candidate with a transverse momentum $\mathrm{p}_{T}>40 \mathrm{GeV}$. Electrons and muons are reconstructed using standard CMS algorithms. Electron candidates must be within the barrel or endcap acceptance regions, with pseudorapidities of $|\eta|<1.442$ and $1.560<|\eta|<2.5$, respectively and have $E_{T}>35 \mathrm{GeV}$. Additionally, an isolation cuts are required to suppress the misidentification of jets as electrons. Muon candidates must be isolated and have $\mathrm{p}_{T}>45 \mathrm{GeV}$, measured with an accuracy of $\delta\left(p_{T}\right) / p_{T}<0.3$. For signal events, the total triggering, reconstruction and identification efficiency is estimated from Monte Carlo simulated events. Each dilepton event is required to have a well reconstructed vertex. The opposite sign of dilepton events is required only for dimuon channel to reject mismeasured muons, as a charge misassignment implies a large momentum measurement error.

To be robust against uncertainties in the absolute background level, the search for resonances is based on a shape analysis of the dilepton mass spectrum. The main SM background is the DrellYan production $\left(\mathrm{Z} / \gamma^{*}\right)$. There are also background contributions from $\mathrm{tt}, \mathrm{tW}$, and diboson production processes. These background components are derived from Monte Carlo simulations. In addition, 


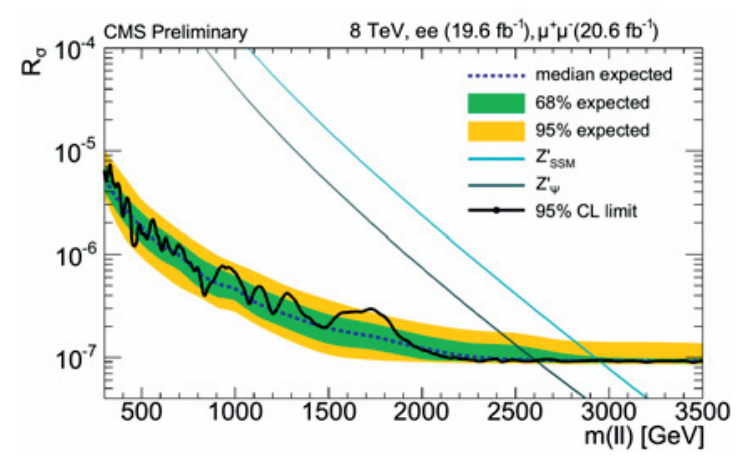

Figure 2. Upper limits from combined dilepton state as a function of resonance mass $M$ on the production ratio $\mathrm{R} \sigma$ of cross section times branching fraction into lepton pairs for $\mathrm{Z}_{S S M}^{\prime}$ and $\mathrm{Z}_{\psi^{\prime}}^{\prime}$ boson production to the same quantity for $\mathrm{Z}$ bosons. Shaded green and yellow bands correspond to the $68 \%$ and $95 \%$ quantiles for the expected limits.

jets may be misidentified as leptons and contribute to the dilepton invariant mass spectrum through multijet and vector boson plus jets final states. The final invariant mass distribution in dimoun and dielectron channels are shown in Fig.1, where the data is compared to the expected backgrounds. No statistically significant excess especially at tails of data is observed. In the absence of a signal, limits are set on the ratio $\mathrm{R}_{\sigma}=\frac{\sigma(p p \rightarrow \mathbf{Z}+X \rightarrow l l+X)}{\sigma(p p \rightarrow \mathbf{Z}+X \rightarrow l l+X)}$ of the production cross section times branching fraction for high-mass resonances to that for the $Z$ boson. The results of the search are interpreted in the context of the Sequential Standard Model which predict narrow $Z_{S S M}^{\prime}$ with SM-like couplings and $Z_{\psi^{\prime}}^{\prime}$ expected in Grand Unified Theories (GUT). The analysis excludes, at $95 \%$ confidence level, $Z_{S S M}^{\prime}$ resonance lighter than $2960 \mathrm{GeV}$ and a GUT-inspired $\mathrm{Z}_{\psi}^{\prime}$ lighter than $2600 \mathrm{GeV}$ for the combined muon and electron channels, as shown in Fig.2.

\subsection{Dijet resonances}

Many extensions of the SM predict the existence of new massive objects that couple to quarks or antiquarks (q) and gluons (g), thus resulting in resonances in the dijet mass spectrum. A search for such resonances decaying into a pair of jets has been performed by the CMS detector using $19.6 \mathrm{fb}^{-1}$ of pp collisions at $8 \mathrm{TeV}$ [4].

Events are preselected using standard jet triggers. The trigger jet candidates from one event are then clustered using the anti-kT algorithm with a cone size parameter $\mathrm{R}=0.5$. New jets with $\mathrm{p}_{T}>40$ $\mathrm{GeV}$ and $|\eta|<3.0$ are used to compute the scalar sum of the jet transverse momenta, $\mathrm{H}_{T}=\Sigma_{i=1}^{N_{j e t}} p_{T}$. The $\mathrm{H}_{T}$ sum above $650 \mathrm{GeV}$ is used for the final event triggering. Selected events must have a reconstructed primary vertex within the range $z<24 \mathrm{~cm}$. All reconstructed object which are identify as muons, electrons, photons, and charged/neutral hadrons are then clustered into jets using the the same configuration as used for the jet reconstruction at the trigger level. Events are selected if at least two jets with $\mathrm{p}_{T}>30 \mathrm{GeV}$ and $|\eta|<2.5$ are present. Two most energetic jets must satisfy the pseudorapidity separations $\Delta \eta_{j j}<1.3$. Next, events are combined into a dijet system consisting of two wide jets build around two leading jets with a cone size $\mathrm{R}_{w}$ ide $=1.1$. The wide jet algorithm is intended to reduce sensitivity to gluon radiation. It was verified by the MC simulation (PYTHIA V6.424, Tune D6T) and CMS detector simulation for a few representative resonance models. Obtained mass invariant spectrum of various dijet system is shown on Fig.3(left). The dijet mass distributions have 

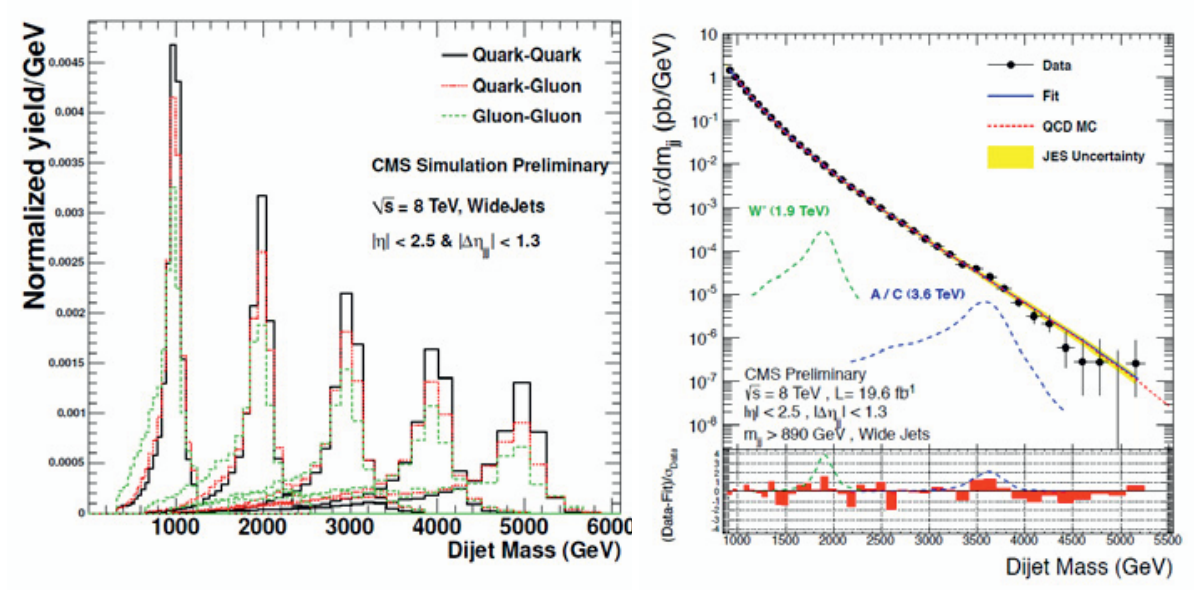

Figure 3. (left) The reconstructed resonance mass spectrum generated with the PYTHIA MC simulation for $\mathrm{qq} \rightarrow \mathrm{G} \rightarrow \mathrm{qq}, \mathrm{qg} \rightarrow \mathrm{q}^{*} \rightarrow \mathrm{qg}, \mathrm{gg} \rightarrow \mathrm{G} \rightarrow \mathrm{gg}$ for resonance masses of 1.0, 2.0, 3.0, 4.0, and 5.0 TeV. (right) Dijet mass spectrum from wide jets (points) compared to a smooth fit (solid) and to predictions including detector simulation of QCD and signal resonances. The error bars are statistical only. The bin-by-bin fit residuals are shown at the bottom.

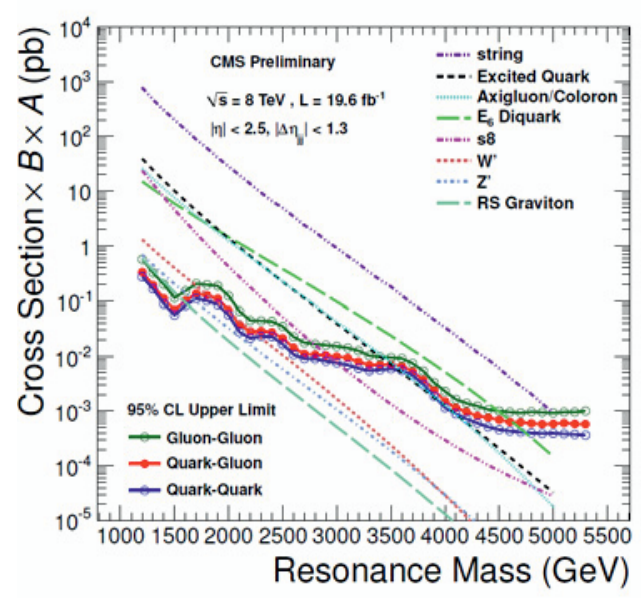

\begin{tabular}{|c|c|}
\hline Final State & $\begin{array}{c}\text { Obs. Mass Excl. } \\
{[\mathrm{TeV}]}\end{array}$ \\
\hline $\mathrm{qg}$ & {$[1.20,5.08]$} \\
$\mathrm{qg}$ & {$[1.20,3.50]$} \\
$\mathrm{qq}$ & {$[1.20,4.75]$} \\
$\mathrm{qq}$ & {$[1.20,3.60]+[3.90,4.08]$} \\
$\mathrm{gg}$ & {$[1.20,2.79]$} \\
$\mathrm{q} \overline{\mathrm{q}}$ & {$[1.20,2.29]$} \\
$\mathrm{q} \overline{\mathrm{q}}$ & {$[1.20,1.68]$} \\
$\mathrm{qq}+\mathrm{gg}$ & {$[1.20,1.58]$} \\
\hline
\end{tabular}

Figure 4. The observed $95 \%$ CL upper limits from the high-mass analysis on $\sigma \times B \times A$ for dijet resonances of the type gluon-gluon, quark-gluon, and quark-quark, compared to theoretical predictions for resonances of various final states as labeled on the plot.

a Gaussian core coming from the jet energy resolution and a tail towards lower mass values coming primarily from QCD radiation. Contributions of the tail depend on the parton content of the resonance (qq, qg, or gg). The spectrum width increases with number of gluons due to the final state radiation and the mass peak is shifted towards lower masses. With the final cut, events with the dijet invariant mass $\mathbf{M}_{j j}>890 \mathrm{GeV}$ are selected and shown in Fig.3(right). The measured dijet mass spectrum is fitted 
with a smooth, empirical parametrization which well describes the prediction from simulated QCD dijet events. No significant difference between the distribution of the data points and the smooth fit through all the data is observed. Therefore, for new physics processes, we set the model-independent upper $95 \%$ confidence level limits on the cross section $(\sigma)$ of new particle production multiplied by the branching fraction $(B)$ to jets and the detector acceptance $(A)$. By comparing these generic limits with theoretical predictions for the production cross section of new particles for several models, the lower limits on the mass can be set for various resonances, as shown in Fig 4. The lower mass limits reach up to $5.1 \mathrm{TeV}$, depending on the model.

\section{Multiparticle signature of Black Holes}

The possibility of production of microscopic black holes in particle collisions has been predicted in models with low scale gravity. The basis of the analysis preformed at the CMS detector is a model proposed by Arkani-Hamed, Dimopoulos, and Dvali (ADD). This model introduce $n$ large, flat, extra spatial dimensions, compactified on an $n$-dimensional torus or a sphere. By opening the multidimensional space only to the gravitational interaction, the fundamental Planck scale in $4+$ $n$ dimensions, $\mathrm{M}_{D}$, is lowered to the electroweak symmetry breaking scale. The enhanced gravity in multidimensional space allows the formation of microscopic black holes (BH). At LHC, if the scale $\mathrm{M}_{D}$ is in the $1 \mathrm{TeV}$ range, partons colliding with energy exceeding scale $\mathrm{M}_{D}$, may collapse into the $\mathrm{BH}$. Once the $\mathrm{BH}$ is produced, it evaporates almost instantaneously by emitting energetic particles. Its characteristic signature is a large number of energetic final-state particles of various types (jets,leptons,photons). Therefore, the aim of searches for BH is to select events with large total transverse energy with multiple energetic jets, leptons, and photons in the final state.

At the CMS experiment, a search [5] for microscopic black holes and string balls has been performed on a data sample of pp collisions at $8 \mathrm{TeV}$ corresponding to an integrated luminosity of $12 \mathrm{fb}^{-1}$.
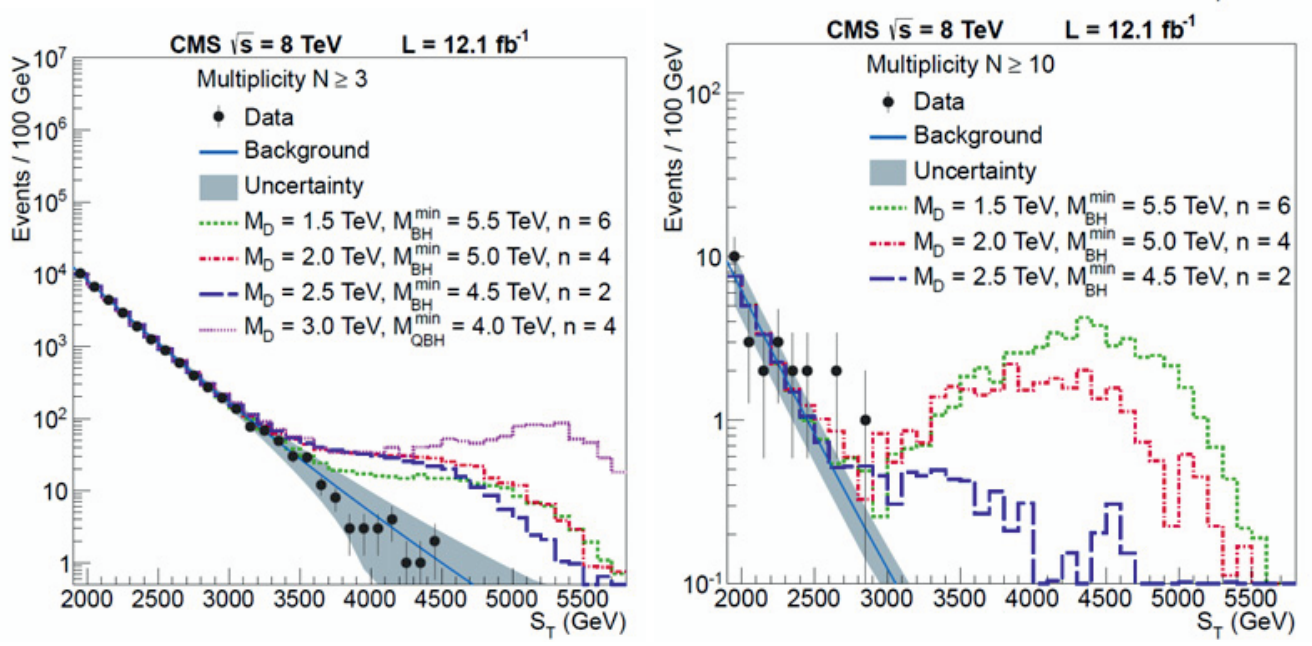

Figure 5. Distribution of the total transverse energy, $\mathrm{S}_{T}$, for events with multiplicity $N \geq 3$ (left) and $N \geq 10$ (right) in the final state. Observed data are represented as points with error bars; the solid line with a shaded band is the multijet background prediction and its systematic uncertainty. The expected black hole signals for three parameter sets are also shown. 
The data used for this analysis are collected with a set of triggers based on the $\mathrm{H}_{T}$ variable. The thresholds for the HT triggers increased from 200 to $750 \mathrm{GeV}$, depending on the data-taking period. Information from all subdetector is used in a standard reconstruction to identify all neutral and charged particles in the event. The missing transverse energy, $M E T$, is defined as the absolute value of the vector sum of transverse momenta of all the objects reconstructed in an event.

The search for black holes is based on a search for a deviation from the SM background predictions in the $\mathrm{S}_{T}$ spectra observed in data. The $\mathrm{S}_{T}$ variable is defined as the scalar sum of transverse momenta of all the final-state objects in the event with $\mathrm{p}_{T}$ in excess of $50 \mathrm{GeV}$. If the MET in the event exceeds $50 \mathrm{GeV}$, its value is also added to the $\mathrm{S}_{T}$ variable. For each event, a multiplicity $N$ is determined from all the objects in the events (excluding MET) that enter the calculation of $\mathrm{S}_{T}$. The SM background is completely dominated by QCD multijet production and is estimated directly from data using a method based on $\mathrm{S}_{T}$ multiplicity invariance. It was empirically observed that the shape of the $\mathrm{S}_{T}$ spectrum is approximately independent of $N$, so the shapes of the $S_{T}$ spectrum for any number of objects can be estimated using a fit to the dijet data (for $N=2$ ). The scaling of the background to higher multiplicities is performed by normalising the background shape to data in each inclusive multiplicity bin in the control range $\left(1.9<\mathrm{S}_{T}<2.3 \mathrm{TeV}\right)$. The final search for BHs is performed for various inclusive multiplicity bins, from $N \geq 2$ to $N \geq 10$. Two of all $N$ distributions are shown in Fig.5. No deviations from the SM background predictions in each of these bins has been found. Therefore, limits on black hole and string ball production rates has been set. With this search, black holes expected in various models with masses below $4.3-6.2 \mathrm{TeV}$ are excluded in the context of a number of benchmark models. Stringent limits on black hole precursors string balls are also set. In Fig.6, selected limits obtained in the analysis are presented.
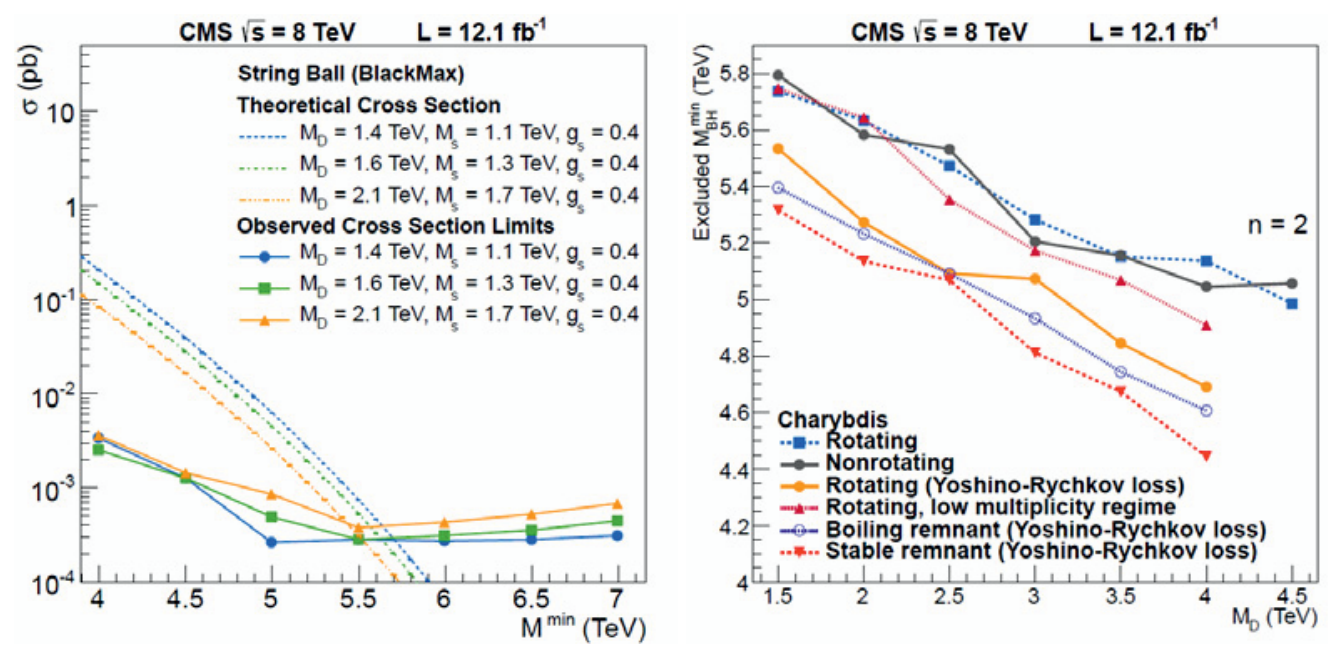

Figure 6. (left) The cross section upper limits at $95 \%$ CL from the counting experiments optimized for various string ball parameter sets (solid lines) compared with predicted signal production cross section (dashed lines) as a function of minimum string ball mass. (right) The $95 \%$ CL lower limits on the semiclassical black hole mass derived from the upper $95 \%$ CL limits on cross section times branching fraction as a function of the fundamental Planck scale $\mathbf{M}_{D}$, for various models. The areas below each curve are excluded by this search. 


\section{Long-lived massive particles}

Many models of new physics predict the existence of massive, long-lived particles (LLP) which manifest themselves through unusual signatures, e.g. kink track or delayed signals. Such scenarios arise in various supersymmetric scenarios[6? ] such as Split SUSY or SUSY with very weak R-parity violation, SUSY with gauge mediated SUSY breaking (GMSB), hidden valley models, and others. Long-lived particles are searched at the CMS detector taking advantage of techniques to measure properties of the track.

\subsection{Long-lived charged particles}

Searches for long-lived charged particles[6] in pp collisions at the centre-of-mass energy 7 and $8 \mathrm{TeV}$ have been performed with the CMS detector with an integrated luminosity of $5.0 \mathrm{fb}^{-1}$ and $18.8 \mathrm{fb}^{-1}$, respectively.

Long-lived, charged particles can have speed $v=\beta \cdot c$ significantly less than the speed of light $c$. With lifetimes greater than a few nanoseconds, these particles can travel distances comparable to the size of modern detectors and thus appear to be stable. These particles are muon-like. They can also have their charge $Q$ not equal to the elementary positive or negative charge $1 e$ or they can be fractionally charged $(Q=n \cdot 1 / 3 e)$ or multiply charged $(Q=n \cdot 1 e)$. Additionally, some LLP may combine with SM particles to form composite objects, such as $R$-hadrons being long-lived SUSY particles, stop (scalar top quark) or gluino after their hadronization. Interactions of these composite objects with the detector may change their constituents and possibly their electric charge, further limiting the ability of standard algorithms to identify them.
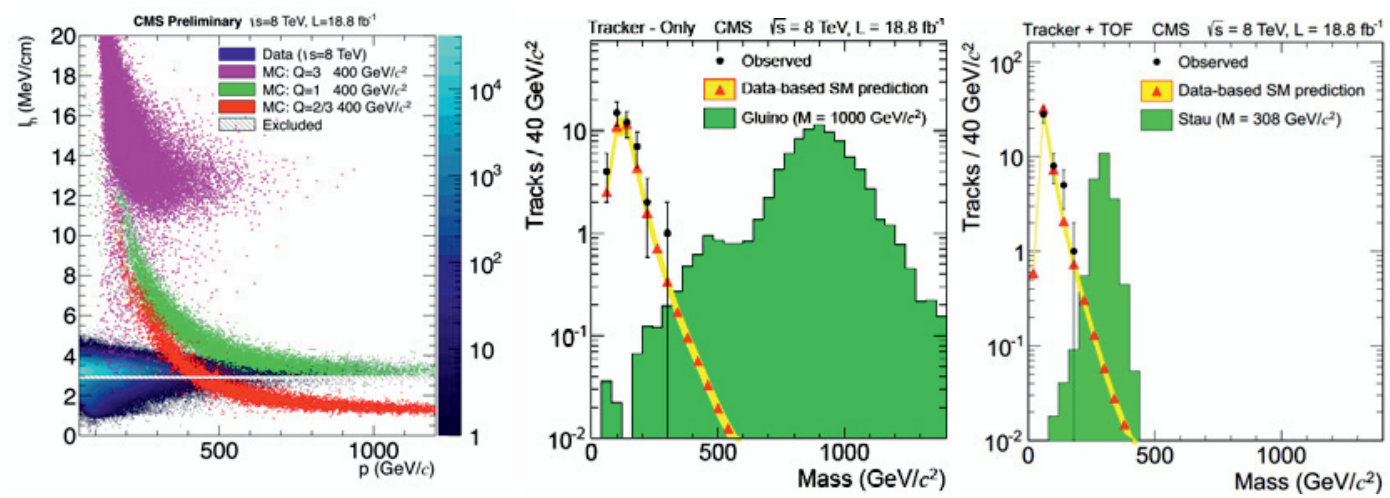

Figure 7. Distribution of a dE/dx estimator, $\mathrm{I}_{h}$, versus particle momentum for $8 \mathrm{TeV}$ data and MC simulated LLP candidates of different charges (left). Observed and predicted mass spectrum for candidates entering the tracker-only (middle) column) or tracker+TOF (right) signal region for the final selection.

Data collected with the CMS detector are used to study the momentum, energy depositions, and time-of-flight (TOF) of signal candidates. These measurements allow for the determination of the particle's mass. First measurement is an estimator $I_{h}$ of the energy deposition DE/dx in the inner tracker; $\mathrm{I}_{h}=K m^{2} / p^{2}+C$, where parameters $K$ and $C$ are determined from data. The expected behaviour of the estimator for data and simulated signal is shown in Fig.7(left). The second measurement is performed in the muon detector using the TOF technique. The particle's velocity $\beta$ parameter is obtained from 

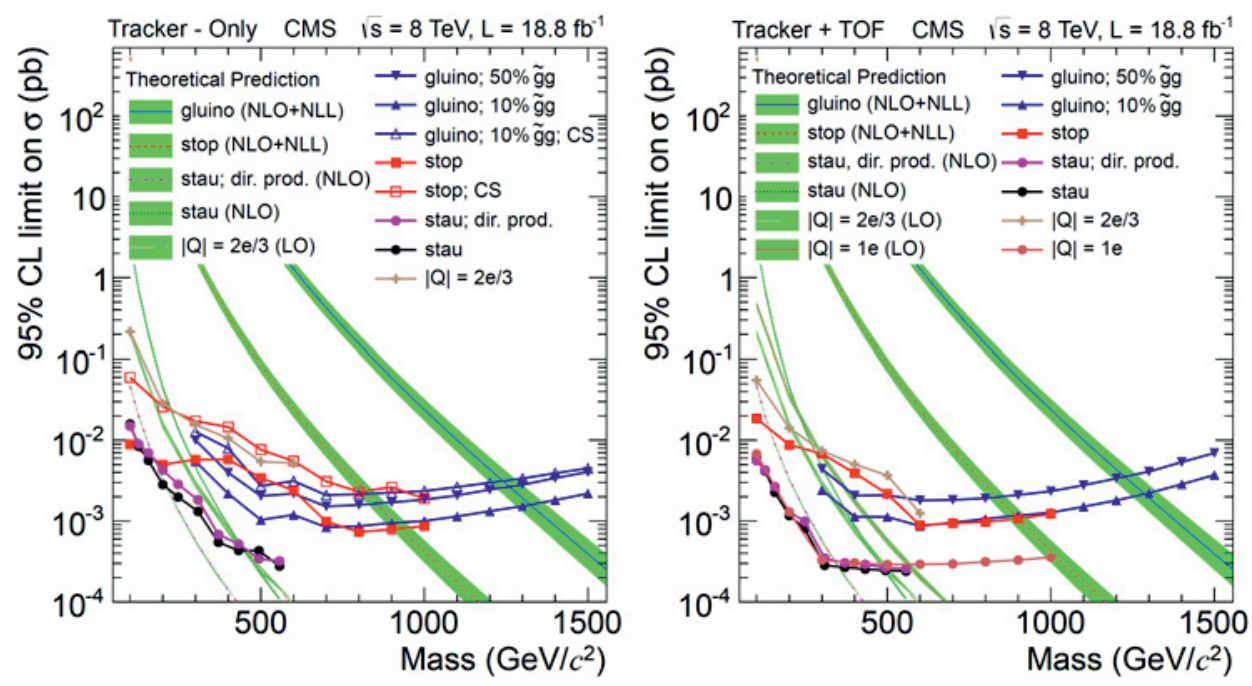

Figure 8. Upper cross section limits at $95 \% \mathrm{CL}$ on various signal models for the tracker-only analysis (left) and tracker+TOF analysis (right) for $8 \mathrm{TeV}$ data.

the formula: $\beta=1+c \delta_{t} / L$, where $\delta_{t}$ is measured time difference of a hit in the muon chamber relative to that expected for a $\beta=1$ particle; $L$ is the flight distance from the interaction point.

Multiple search strategies are used to separate signal from background depending on the nature of the LLP under investigation. Analysis use various combinations of signatures in the inner tracker only, inner tracker and muon detector (with TOF), and muon detector only in five following paths: (i) for charged LLP: tracker+TOF are used to measure: $\mathrm{p}_{T}, \mathrm{DE} / \mathrm{dx}$ and $\beta$; (ii) for becoming neutral LLP: tracker-only is used to measure: $\mathrm{p}_{T}, \mathrm{DE} / \mathrm{dx}$; (iii) for neutral LLP in the tracker, becoming charged: muon-only to measure $\mathrm{p}_{T}, \beta$; (iv) for fractionally charged LLP: tracker-only to measure: $\mathrm{p}_{T}$, $\mathrm{DE} / \mathrm{dx}$ which should be smaller then for SM particles; (v) for multiply charged LLP: tracker+TOF to measure: $\mathrm{DE} / \mathrm{dx}, \beta$; , do not use $\mathrm{p}_{T}$, because algorithms to reconstruct momentum work only for singly charged particles.

All events must pass a trigger requiring either the reconstruction of a muon with high $\mathrm{p}_{T}>40$ $\mathrm{GeV}$ or large missing transverse energy, MET $>150 \mathrm{GeV}$. The first level of muon trigger allows for late arriving particles by accepting tracks that produce signals in the muon system subdetector, Resistive Plate Chambers, within either the $25 \mathrm{~ns}$ time window corresponding to the interaction bunch crossing or the following $25 \mathrm{~ns}$ time window. For the data used in this analysis, the second $25 \mathrm{~ns}$ time window of pp collisions is empty because of the $50 \mathrm{~ns}$ LHC bunch spacing during the 2011 and 2012 operation. Preselection criteria on the inner tracker track and the muon track allow to select well reconstructed object. For all of the analyses, results are based upon a comparison of the number of candidates passing the final section criteria with the number of background events estimated from the numbers of events that fail combinations of the criteria. The background expectation in the signal region, $\mathrm{D}$, is estimated as $\mathrm{D}=\mathrm{BC} / \mathrm{A}$, where $\mathrm{B}(\mathrm{C})$ is the number of candidates that fail the first (second) criteria but pass the other one and $\mathrm{A}$ is the number of candidates that fail both criteria. The method works if the probability for a background candidate to pass one of the criteria is not correlated with whether it passes the other criteria. For various analysis, two or three criteria are used in $\mathrm{ABCD}$ 

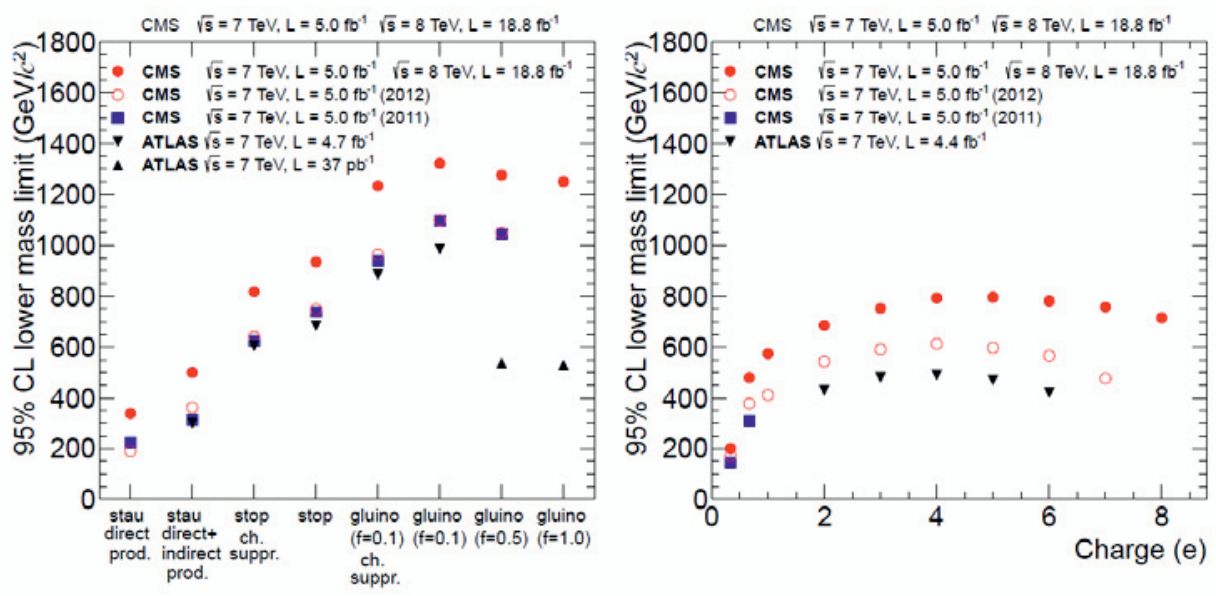

Figure 9. Lower mass limits at $95 \% \mathrm{CL}$ for various models compared with previously published results. The model type is given on the $\mathrm{x}$-axis (left). Mass limits are shown for Drell-Yan like production of fractionally, singly, and multiply charged particles (right).

method, $\mathrm{p}_{T}$ and $1 \beta$ or $\mathrm{DE} / \mathrm{dx}$ discriminator. For each analysis, fixed selections on the appropriate set of two $\mathrm{DE} / \mathrm{dx}$ discriminators, $\mathrm{p}_{T}$, and $1 \beta$ are used to define the final signal region (and the regions for the background prediction). These values are chosen to give discovery potential over the signal mass regions of interest. For the tracker-only and tracker+TOF analyses, an additional requirement on the reconstructed mass is applied. Fig.7 shows the observed and predicted mass distributions for the tracker-only and tracker+TOF analyses with the final selection. The bump at lower mass values expected from the signal MC is due to the saturation of the strip electronic readout. The data are consistent with the expected background, and upper limits are set on the production cross section of long-lived gluinos, stops, and staus (scalar tau leptons), as well as pair produced long-lived leptons. The $8 \mathrm{TeV}$ results are shown in Fig.8. The tracker-only analysis excludes two kind of gluino masses below 1322 and $1233 \mathrm{GeV}$. Stop masses below 935 (818) GeV are excluded for the cloud (chargesuppressed) models. In addition, the tracker+TOF analysis excludes stau $t \tilde{a} u_{1}$ masses below 500 (339) $\mathrm{GeV}$ for the direct+indirect (direct only) production. Drell-Yan signals with $Q=2 e / 3$ and $Q=1 e$ are excluded below 220 and $574 \mathrm{GeV}$ respectively. The summary of all exclusion mass limits is presented in Fig.9. These limits are the most stringent to date.

\section{Conclusions}

The CMS Collaboration has performed a wide range of searches for new particles, exploiting different signatures and using various detection techniques. Despite this huge effort, no exotic signal has been observed in the data. Thus, exclusion limits on cross-sections and masses, or model-independent, limits, are given for various models. Since low-mass supersymmetry has not been found either, the theoretical prejudice that Nature could be exotic is getting stronger. Thats what makes searches for exotic physics so exciting, and at the same time challenging. Consequently, even more effort will be put into searches for exotic physics in the next run of the LHC, starting in 2015 at a center-of-mass energy of $13 \mathrm{TeV}$. 
EPJ Web of Conferences

\subsection{Acknowledgment}

This work was supported in part by the Polish Ministry of Science and Higher Education and National Science Centre grant N N202 167440.

\section{References}

[1] H. Baer, X. Tata, Weak Scale Supersymmetry, ISBN-10:0521857864, CUP (2006)

[2] CMS Collaboration, The CMS experiment at the CERN LHC, JINST,3:S08004 (2008)

[3] CMS Collaboration, Search for Narrow Resonances in Dilepton Mass Spectra in pp Collisions at $\sqrt{s}=8 \mathrm{TeV}$, CMS-PAS-EXO-12-061 (2012)

[4] CMS Collaboration, Search for Narrow Resonances using the Dijet Mass Spectrum with 19.6 $\mathrm{fb}^{-1}$ of pp Collisions at $\sqrt{\mathrm{s}}=8 \mathrm{TeV}$, CMS-PAS-EXO-12-059 (2012)

[5] CMS Collaboration, Search for microscopic black holes in pp collisions at sqrt $(s)=8 \mathrm{TeV}$, 10.1007/JHEP07(2013) 178

[6] CMS Collaboration, Searches for long-lived charged particles in pp collisions at sqrt $(s)=7$ and $8 \mathrm{TeV}, 10.1007 / \mathbf{J H E P 0 7 ( 2 0 1 3 ) 1 2 2}$ 